\title{
ERCC1 polymorphism predicts clinical outcomes of oxaliplatin-based chemotherapies in advanced colorectal cancer: A systemic review and meta- analysis.
}

\author{
Xue-Qiong Han, Tao Ren, Wei-Dong Yi, Ming-Hui Sun, Lu Zhou, Jian-Xun Lu* \\ Department of Medical Oncology, The First People's Hospital of Nanning, Nanning, Guangxi 530022, People's \\ Republic of China
}

\begin{abstract}
Objective: To systematically evaluate the precise role of the excision repair cross-complementing group 1 (ERCC1) polymorphism Asn118Asn (C19007T, rs11615) in curative effects and prognosis of patients with advanced colorectal cancer (CRC) receiving oxaliplatin-based chemotherapy.

Methods: Qualified studies were retrieved from databases including PubMed, EMBASE, Wanfang and CNKI until January 31 ${ }^{\text {st }}$, 2015. Literature published in English and Chinese were selected for metaanalysis on relationship between ERCC1 polymorphism Asn118Asn and therapeutic effects and prognosis of advanced CRC patients receiving oxaliplatin-based chemotherapy. Data extracted from these articles included study ID, title, author, publication year, ethnicity, country, numbers of cases and controls, progression-free survival (PFS) and overall survival (OS). Pooled odds ratios (ORs) and their 95\% confidence intervals (CIs) were used to estimate objective response with hazard ratios (HRs) using Stata software (version 11.0).

Results: A total of 13 articles were included in the study. Overall, meta-analysis showed no significant correlation between the ERCC1 C118T polymorphism and objective response $(\mathrm{OR}=0.78 ; 95 \% \mathrm{CI}$ for 0.29-2.07), PFS $(\mathrm{HR}=1.70,95 \% \mathrm{CI}=\mathbf{0 . 9 2 - 3 . 1 4})$ or OS $(\mathrm{HR}=1.69,95 \% \mathrm{CI}=\mathbf{0 . 9 1 - 3 . 1 4})$ in advanced CRC patients with oxaliplatin-based chemotherapy. Stratified analysis among Asian and Caucasian populations showed that the ERCC1 C118T polymorphism was not significantly correlated with objective response $(\mathrm{OR}=2.03 \mathrm{vs} .0 .40 ; 95 \% \mathrm{CI}=0.62-6.68 \mathrm{vs}$. $0.12-1.30)$, but was significantly correlated with PFS and OS. Moreover, Asian patients carrying T/T or T/C genotypes of ERCC1 C118T had significant shorter PFS (HR=2.41, 95\% CI=1.86-3.11) and $O S(H R=2.36,95 \% C I=1.76-3.16)$.

Conclusion: In patients with advanced CRC undergoing oxaliplatin-based chemotherapy, there is no correlation between ERCC1 Asn118Asn (C/T) gene polymorphism and therapeutic effects. Asian patients with $\mathrm{T}$ allele has shorter PFS and OS.
\end{abstract}

Keywords: ERCC1, Colorectal cancer, Single nucleotide polymorphism (SNP), Chemotherapy, Meta-analysis. Accepted on April 26, 2018

\section{Introduction}

Colorectal cancer $(\mathrm{CRC})$ is the third most common malignancies in the world [1], with increasing morbidity and mortality [2,3]. Currently the most effective treatment for colorectal cancer is still surgery. However, after radical resection a large number of patients appear in situ recurrence and distant metastasis. Moreover, some patients are in advanced stage at diagnosis and lose the opportunity for radical resection [4,5]. Various studies have currently confirmed that the chemotherapy can improve the quality of life and prolong the survival time of CRC patients. The national comprehensive cancer network (NCCN) has proposed oxaliplatin combined with 5-fluorouracil as standard first line treatment guidelines for colorectal cancer therapy [6].
CRC patients showed different reactions with the same intensity of chemotherapy drugs, and some patients do not benefit from chemotherapy. Moreover, a considerable portion of patients with oxaliplatin based chemotherapy appear different degree of peripheral neurotoxicity. Therefore, if the clinical curative effect of oxaliplatin could be predicted, clinicians can choose a chemotherapy regimen with high efficiency and low toxicity according to the inspection results.

Oxaliplatin (L-OHP) is the third generation platinum compounds, which kills tumor cells by blocking DNA replication and transcription [7,8]. Excision repair crosscomplementing group 1 (ERCC1) is an important DNA repair gene and a marker gene of nucleotide excision repair (NER) activity [9]. The NER is a major pathway of DNA damage repair and ERCC1 is an important rate limiting enzyme of 
NER process. Various studies showed that single nucleotide polymorphisms (SNP) in ERCC1 encoding the 118 codon (Asn118Asn, C19007T, rs11615) change AAC-AAT can affect DNA damage repair capacity [10-13]. Therefore, genetic variation at this gene locus may affect the individual sensitivity to chemotherapeutic drugs [10].

Although the ERCC1 Asn118Asn function is not completely clear, it is confirmed that polymorphism of this locus might be associated with efficacy and prognosis by platinum drugs $[14,15]$, with some controversy between the study results. Therefore, this article utilized the meta-analysis to make comprehensive evaluation on association between ERCC1 Asn118Asn polymorphism and chemotherapeutic effects in advanced CRC patients receiving oxaliplatin based chemotherapy.

\section{Materials and Methods}

\section{Inclusion criteria}

- Studies on ERCC1 Asn118Asn gene polymorphism and colorectal cancer by oxaliplatin chemotherapy efficacy or prognosis.

- The original literatures published in the full text.

- The article provides enough information, such as the effect odds ratio (OR) and 95\% confidence interval (CI) in each genotype, or the OR and $95 \% \mathrm{CI}$ could be calculated from the data.

- The distribution of the genotypes in the literature conformed to the Hardy-Weinberg equilibrium.

\section{Exclusion criteria}

- The data were not complete, or repeated literatures.

- Literatures were case report, literature review or animal experiment.

- The use of other languages except Chinese or English.

\section{Literature search strategy}

Literatures were searched on published databases of PubMed, EMBASE, web of science, CNKI, VIP, Wanfang and CBM. Meanwhile, literatures were also manually searched on references of reviews. The English retrieval words were used for searching as following: ERCC1 or excision repair crosscomplementing group 1; colon or colorectal cancer; polymorphism or variant; treatment or chemotherapy. The retrieval time was up to January 31, 2015.

\section{Data extraction}

After the included studies were determined, two authors were appointed to read text and extract data information from each included study, including title, author, publication year, study countries, the race of study subject and the number of cases.

\section{Statistical analysis}

The evaluation on the therapeutic effects of chemotherapy is made according to the World Health Organization (who) standards or the response evaluation criteria in solid tumors (RECIST 1.0) [16], and is mainly divided into the following categories: complete remission $(\mathrm{CR})$, partial remission (PR), stable disease (SD) and progress disease (PD). In this study we defined the efficacy as response $(\mathrm{PR}+\mathrm{CR})$ and non-response $(\mathrm{SD}+\mathrm{PD})$. The genetic data were extracted and the hazard ratios (HR) and 95\% CI in each literature were pooled and analyzed for prognosis.

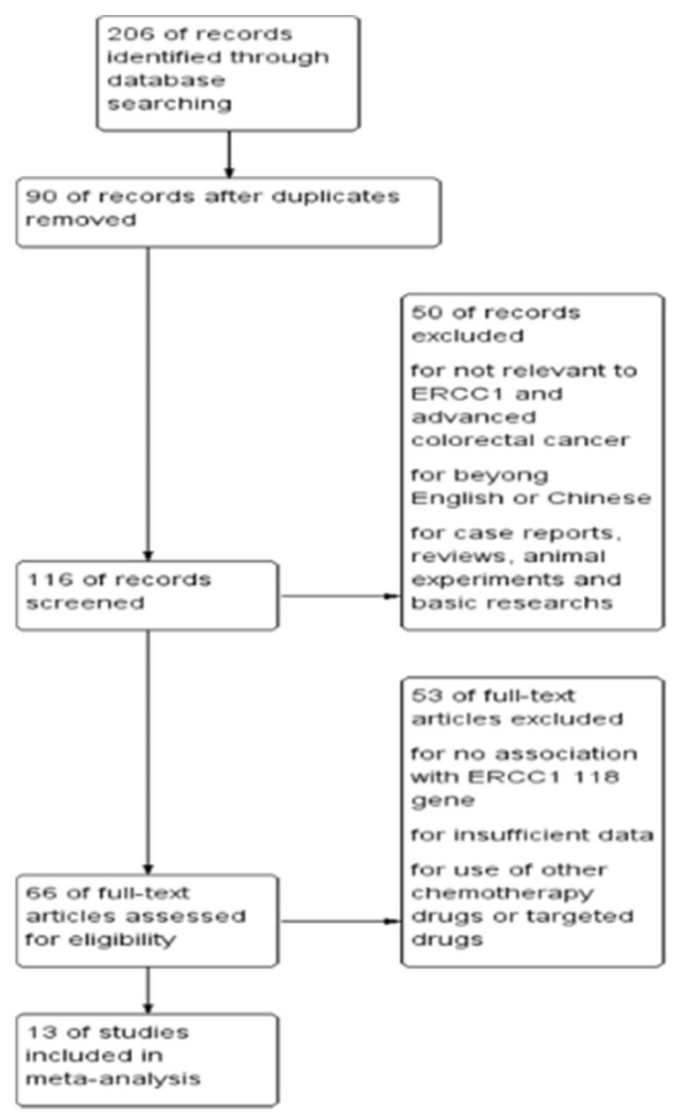

Figure 1. Flow chart of literature search.

Stata 11.0 software was used to perform the meta-analysis Pooled analysis was performed by dominant model on the association between ERCC1 gene polymorphism and effect and prognosis of oxaliplatin-based chemotherapy. Subgroup analysis was performed within different races.

The heterogeneity between studies was assessed by Cochran's $\mathrm{Q}$ test and inconsistency index $\mathrm{I} 2$ test method. The size of heterogeneity was assessed by $\mathrm{I} 2$. When $\mathrm{I} 2<50 \%$, the fixedeffect model was used to combine the data; otherwise the random-effect model was used to combine the data.

The funnel plot was drawn to judge publication bias by Begger method. The included literature therapy was evaluated by Newcastle-Ottawa scale (NOS). 


\section{Results}

\section{Literature selection and inclusion}

A total of 206 literatures were detected, and 90 repeated literatures were excluded. In the remaining 116 articles, after viewing their titles and abstracts, 50 articles were excluded due to non ERCC1 related research in and advanced CRC and nonEnglish or Chinese articles. We read the full text of the remaining 66 articles, and 53 articles were excluded due to incomplete data or use of other chemotherapy drugs. Ultimately 13 literatures were included into meta-analysis (Figure 1), and the basic characteristics of the literature are shown in Table 1.

\section{Meta-analysis}

Meta-analysis was performed on the association between ERCC1 Asn118Asn gene polymorphism and effective rate of oxaliplatin in advanced CRC. Heterogeneity test showed that I2 value $>75 \%$, and the random-effect model was used to calculate the combined effect value. A total of 9 articles were included, among which 4 articles were analyzed for effect values by merging the CT and TT values, while 5 articles were analyzed for effect values in TT vs. CT $(\mathrm{OR}=0.91,95 \% \mathrm{CI}$ $0.59 \sim 1.55)$, with no statistical significance between the two groups. However, there were statistically significant differences between two groups in effect values co-dominant model TT vs $\mathrm{CC}(\mathrm{OR}=3.08,95 \% \mathrm{CI}=0.898 \sim 10.533, \mathrm{P}=0.030)$ and the dominant model $\mathrm{CT} / \mathrm{TT}$ vs $\mathrm{CC}(\mathrm{OR}=2.403,95 \%$ $\mathrm{CI}=0.975 \sim 5.927, \quad \mathrm{P}=0.042)$. Therefore, $\mathrm{T}$ allele shows dominant inheritance in platinum chemotherapy sensitivity of patients with advanced CRC. Combined test was further performed on dominant genetic model $\mathrm{CC}+\mathrm{TT}$ vs. $\mathrm{CC}$, and no statistically significantly difference was found $(\mathrm{OR}=0.777$, 95\% $\mathrm{CI}=0.292 \sim 2.068, \mathrm{P}=0.613$ ).

Table 1. General characteristics of included literatures.

\begin{tabular}{|c|c|c|c|c|c|c|c|}
\hline Author & Year & Country & Ethnicity & Tumor-type & Cases & Treatment & Outcomes \\
\hline Viguier [17] & 2005 & France & Caucasian & colorectal & 61 & FUOX & TR \\
\hline Ruzzo [18] & 2006 & Italian & Caucasian & colorectal & 175 & $5-F U / C F$ & TR PFS \\
\hline Pare [19] & 2008 & spain & Caucasian & colorectal & 126 & FUOX & TR PFS OS \\
\hline Lianghua [20] & 2008 & China & Asian & colorectal & 62 & FOLFOX & TR PFS \\
\hline Martinez [21] & 2008 & Spain & Caucasian & colorectal & 96 & XELOX & PFS \\
\hline Spindler [22] & 2009 & Denmark & Caucasian & colorectal & 66 & XELOX & TR \\
\hline Chua [23] & 2009 & Australia & Caucasian & colorectal & 115 & FOLFOX & TR,PFS,OS \\
\hline Chang [24] & 2009 & China & Asian & colorectal & 168 & FOLFOX & TR PFS OS \\
\hline Chen [25] & 2010 & China & Asian & colorectal & 166 & FOLFOX & TR OS \\
\hline Liang [26] & 2010 & China & Asian & colorectal & 113 & XELOX/ FOLFOX & PFS \\
\hline Haina Chai [27] & 2012 & China & Asian & colorectal & 64 & FOLFOX & TR \\
\hline Hewenxing [28] & 2012 & China & Asian & colorectal & 73 & FOLFOX & TR PFS OS \\
\hline Lieke [29] & 2013 & Ausaralia & Caucasian & colorectal & 145 & XELOX/FOLFOX & PFS OS \\
\hline
\end{tabular}

Meta-analysis showed that in the dominant model there was no significant correlation between advanced CRC patients carrying $\mathrm{T}$ allele and platinum chemotherapy efficiency $(\mathrm{OR}=0.78, \quad 95 \% \quad \mathrm{CI}=0.29-2.07), \quad \mathrm{PFS} \quad(\mathrm{HR}=1.70, \quad 95 \%$ $\mathrm{CI}=0.92-3.14)$ or $\mathrm{OS}(\mathrm{HR}=1.69,95 \% \mathrm{CI}=0.91-3.14)$.

\section{Subgroup analysis}

This study was analyzed according to the ethnicity of subjects of included literatures. The results showed that in the dominant model there were no significant correlation in Caucasian and Asian populations between advanced CRC patients carrying $\mathrm{T}$ allele in ERCC1 polymorphism Asn 118Asn and the efficacy of oxaliplatin chemotherapy $(\mathrm{OR}=2.03$ vs. $0.40, \quad 95 \%$ $\mathrm{CI}=0.62-6.68$ vs. $0.12-1.30$ ).
In the dominant model, the Asian population carrying $\mathrm{T}$ allele showed a poor PFS $(\mathrm{HR}=2.41,95 \% \mathrm{CI}=1.86-3.11)$, while the Caucasian population showed a good PFS (HR=1.43, 95\% $\mathrm{CI}=0.57-3.59)$. Similarly, the Asian population with the $\mathrm{T}$ allele showed a poor $\mathrm{OS}(\mathrm{HR}=2.36,95 \% \mathrm{CI}=1.76-3.16)$, while the Caucasian population was the opposite $(\mathrm{HR}=1.17,95 \%$ $\mathrm{CI}=0.32-4.30$ ) (Figures 2 and 3 ).

\section{Publication bias analysis}

Begg rank correlation test was performed using Begg's funnel plot, and showed different degree of publication bias in chemotherapy sensitivity analysis, PFS and OS, which was associated with choice of publishing house and the author's subjective research direction (Figure 4). 


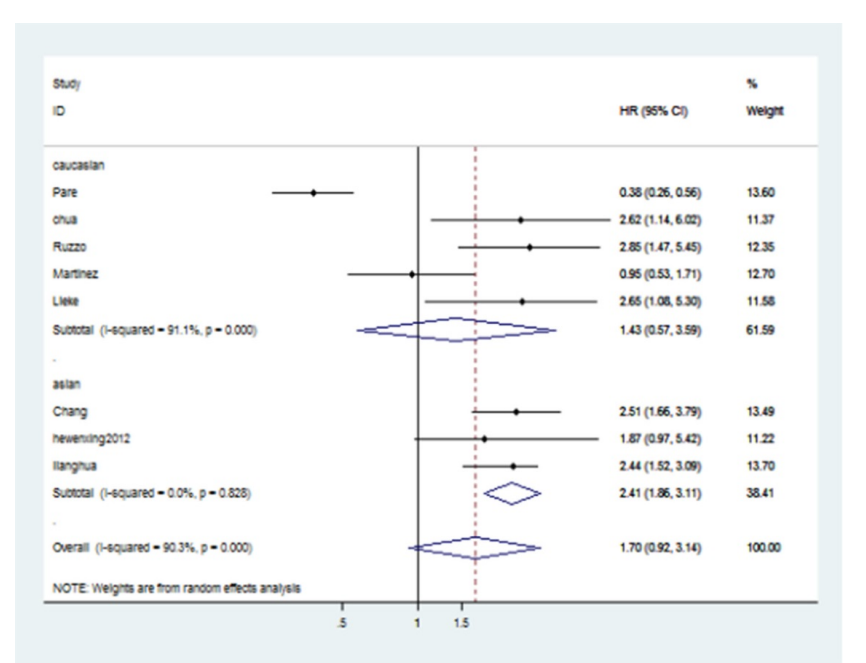

Figure 2. Forest plot of subgroup analysis by ethnicity on the association between ERCC1 C118T polymorphism and PFS of advanced CRC patients with oxaliplatin-based chemotherapy (CT + TT vs. CC). PFS, progression-free survival; CRC, colorectal cancer.

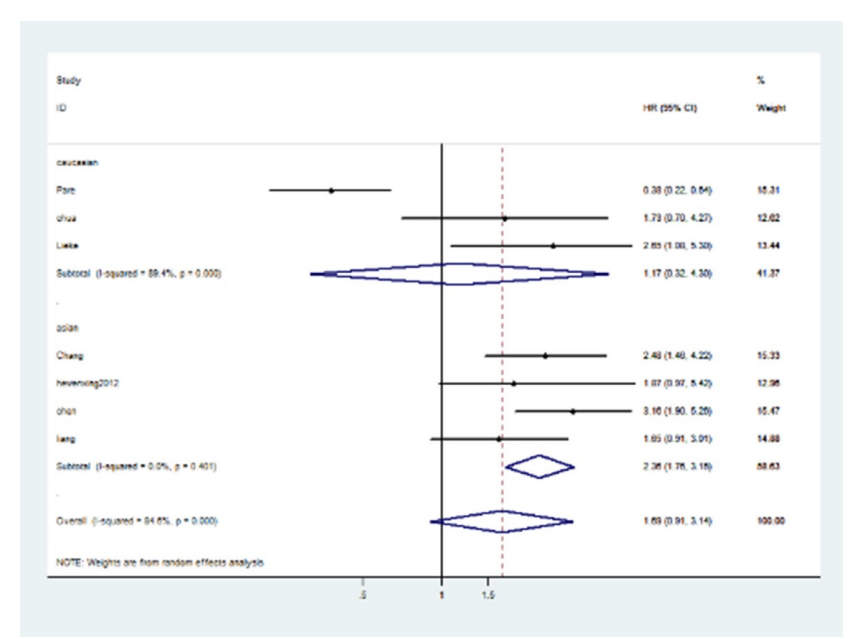

Figure 3. Forest plot of subgroup analysis by ethnicity on the association between ERCC1 C118T polymorphism and OS of advanced CRC patients with oxaliplatin-based chemotherapy (CT $+T T$ vs. CC). OS, overall survival; CRC, colorectal cancer.

\section{Sensitivity analysis}

Meta-analysis was performed after excluding each literature step by step, and the pooled results showed no significant difference, suggesting stability of the included literature.

\section{Literature quality assessment}

The literature quality of non-randomized control study was evaluated using standard as follows: 1) Case control research quality evaluation designed by Lichtenstein MJ;2) Newcastle Ottawa scale (NOS) quantity table of literature quality evaluation. In this study, we used case-control study part of NOS quantity table, and evaluation was made mainly base on diagnostic criteria, representative of included cases, selection and definition of control group, comparable property between two groups and recording method of exposure event. The included literature was evaluated and showed good quality.

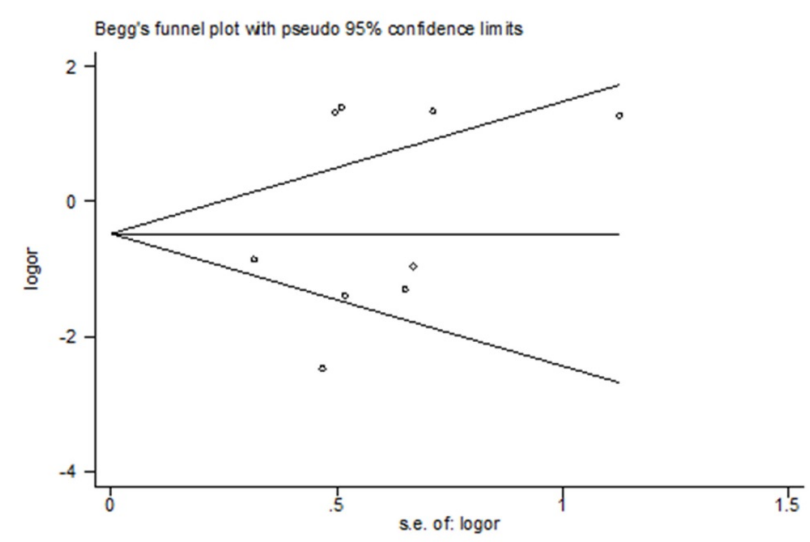

Figure 4. Begg's funnel plot for publication bias of objective response following oxaliplatin-based chemotherapy in advanced CRC by ERCC1 codon 118 polymorphism.

\section{Discussion}

\section{Summary of meta-analysis}

Currently studies are rarely reported on the relationship between ERCC1 gene polymorphism and platinum based chemotherapy sensitivity. Moreover, SNP at 118 codon of ERCC1 gene has been confirmed correlation with ERCC1 mRNA and protein expression levels [8,24]. Studied have been carried out to assess effect of oxaliplatin chemotherapy in advanced CRC patients carrying ERCC1 118 codon SNP. However, it is unclear whether this polymorphism can change ERCC1 protein expression level or capability of DNA repair.

In this study, clinical efficacy rate $(\mathrm{PR}+\mathrm{CR})$ was used as the main index to evaluate chemotherapy. The meta-analysis results showed no correlation between Asn118Asn polymorphism and chemotherapy efficiency, PFS or OS of advanced CRC patients, which was consistent with the results of one previous meta-analysis [30]. However, recently a metaanalysis has reported correlations of ERCC1 Asn118Asn polymorphism with PFS and OS [31], and our results did not confirm it. In fact, our study also contains some advantages. Previous meta-analysis was mostly based on the evaluation of the clinical efficacy of gastric and colon cancer. Considering the heterogeneity of gastric and colon cancer and different biological activity and pathological types, our study selected advanced colorectal cancer as the research subject and made more precise and comprehensive study on chemotherapy efficiency of oxaliplatin.

\section{Subgroup analysis}

Subgroup analysis was performed mainly according to the different races. In Asian population, ERCC1 Asn118Asn polymorphism was correlated with PFS and OS of advanced CRC patients, and patients carrying the $\mathrm{T}$ allele had significantly shorter PFS and OS. The Caucasian populations 
showed the opposite results, with significantly longer PFS and OS in patients carrying the $\mathrm{T}$ allele. The results of subgroup analyses were consistent with the results of previous studies [31].

Among included literatures evaluating effectiveness of chemotherapy, 6 literatures reported the correlation between ERCC1 Asn118Asn gene polymorphism and effective rate of platinum based chemotherapy, and reduced sensitivity to oxaliplatin was reported in CRC patients carrying $\mathrm{T}$ allele (4 literatures) or $\mathrm{CC}$ genotype (2 literatures). The remaining 3 literatures reported no correlation between ERCC1 gene polymorphism and platinum based chemotherapy sensitivity. Further analysis found that in 4 literatures showing correlation between $\mathrm{T}$ allele and reduced chemotherapy efficacy, the included cases from 3 literatures were the Asian population, while in 2 literatures showing correlation between wild homozygote CC genotype and reduced chemotherapy efficacy, the included cases were Asian population and Caucasian population, respectively. The differences between the results of various studies were related to races, interactions of the environment, interactions among genes and the complex DNA repair pathways. This study cannot perform unified screening on features and pathological stage of included cases. Moreover, oxaliplatin was the primary chemotherapy of the first-line chemotherapy for colorectal cancer, and no strict demands were made on the drug compatibility, usage, dosage of fluorouracil. Therefore, confounding factors among drug compatibility, usage and curative effect cannot be ruled out.

\section{Limitations of meta-analysis}

There was still some limitations in this study due to small sample size, as shown in following: 1) Selection bias. This study included only the published literature in English or Chinese, and conference or summary literatures were excluded. Literatures which used targeted or other treatments were also excluded; 2) The overall sample size was small and could not represent the overall population; 3) Language bias. Literatures using other languages besides English and Chinese were not included in this study; 4) Different chemotherapy regimens and detection methods may have certain impact on the outcome of the study; 5) Many literatures with negative results cannot be retrieved in our study.

In conclusion, ERCC1 Asn118Asn gene polymorphism may be correlated with cancer platinum class about chemotherapeutic effect and prognosis of platinum drugs in advanced CRC patients. Due to small sample size, the results of this study need to be confirmed by large sample and high quality clinical investigation.

\section{References}

1. Torre LA, Bray F, Siegel RL, Ferlay J, Lortet-Tieulent J, Jemal A. Global cancer statistics, 2012. CA Cancer J Clin 2015; 65: 87-108.

2. Chen Q, Liu ZC, Cheng LP, Song GH, Sun XB, Zheng RS, Zhang SW, Chen W Q. An Analysis of Incidence and
Mortality of Colorectal Cancer in China, 2003-2007. China Cancer 2012; 3: 179-182.

3. Chen WQ, ZHeng RS, Zeng HM, ZHou HM, Zhang SW, He J. Report of cancer incidence and mortality in China 2011. China Cancer 2015; 1: 1-10.

4. Park DJ, Lenz HJ. Determinants of chemosensitivity in gastric cancer. Curr Opin Pharmacol 2006; 6: 337-344.

5. Ciaparrone M, Quirino M, Schinzari G, Zannoni G, Corsi DC, Vecchio FM, Cassano A, La Torre G, Barone C. Predictive role of thymidylate synthase, dihydropyrimidine dehydrogenase and thymidine phosphorylase expression in colorectal cancer patients receiving adjuvant 5-fluorouracil. Oncology 2006; 70: 366-377.

6. National Comprehensive Cancer Network. Clinical Practice Guedelines in Oncology-Colon Cancer 2015. NCCN, 2015.

7. Lee JH, Gaddameedhi S, Ozturk N, Ye R, Sancar A. DNA damage-specific control of cell death by cryptochrome in p53-mutant ras-transformed cells. Cancer Res 2013; 73: 785-791.

8. Reed E. ERCC1 and clinical resistance to platinum-based therapy. Clin Cancer Res 2005; 11: 6100-6102.

9. Ziani S, Nagy Z, Alekseev S, Soutoglou E, Egly JM, Coin F. Sequential and ordered assembly of a large DNA repair complex on undamaged chromatin. J Cell Biol 2014; 206: 589-598.

10. Yu JJ, Lee KB, Mu C, Li Q, Abernathy TV, BostickBruton F, Reed E. Comparison of two human ovarian carcinoma cell lines (A2780/CP70 and MCAS) that are equally resistant to platinum, but differ at codon 118 of the ERCC1 gene. Int J Oncol 2000; 16: 555-560.

11. Lunn RM, Helzlsouer KJ, Parshad R, Umbach DM, Harris EL, Sanford KK, Bell DA. XPD polymorphisms: effects on DNA repair proficiency. Carcinogenesis 2000; 21: 551-555.

12. Duell EJ, Wiencke JK, Cheng TJ, Varkonyi A, Zuo ZF, Ashok TD, Mark EJ, Wain JC, Christiani DC, Kelsey KT. Polymorphisms in the DNA repair genes XRCC1 and ERCC2 and biomarkers of DNA damage in human blood mononuclear cells. Carcinogenesis 2000; 21: 965-971.

13. Vilmar A, Sorensen JB. Excision repair crosscomplementation group 1 (ERCC1) in platinum-based treatment of non-small cell lung cancer with special emphasis on carboplatin: A review of current literature. Lung Cancer 2009; 64: 131-139.

14. Mlak R, Krawczyk P, Ramlau R, Kalinka-Warzocha E, Wasylecka-Morawiec M, Wojas-Krawczyk K, Kucharczyk T, Homa I, Kozioł P, Ciesielka M, Chudziak D, Milanowski J. Predictive value of ERCC1 and RRM1 gene single-nucleotide polymorphisms for first-line platinum- and gemcitabine-based chemotherapy in non-small cell lung cancer patients. Oncol Rep 2013; 30: 2385-2398.

15. Rumiato E, Cavallin F, Boldrin E, Cagol M, Alfieri R, Basso D, Castoro C, Ancona E, Amadori A, Ruol A, 
Saggioro D. ERCC1 C8092A (rs3212986) polymorphism as a predictive marker in esophageal cancer patients treated with cisplatin/5-FU-based neoadjuvant therapy. Pharmacogenet Genom 2013; 23: 597-604.

16. Therasse P, Arbuck SG, Eisenhauer EA, Wanders J, Kaplan RS, Rubinstein L, Verweij J, Van Glabbeke M, van Oosterom AT, Christian MC, Gwyther SG. New guidelines to evaluate the response to treatment in solid tumors. European Organization for Research and Treatment of Cancer, National Cancer Institute of the United States, National Cancer Institute of Canada. J Natl Cancer Inst 2000; 92: 205-216.

17. Viguier J, Boige V, Miquel C, Pocard M, Giraudeau B, Sabourin JC, Ducreux M, Sarasin A, Praz F. ERCC1 codon 118 polymorphism is a predictive factor for the tumor response to oxaliplatin/5-fluorouracil combination chemotherapy in patients with advanced colorectal cancer. Clin Cancer Res 2005; 11: 6212-6217.

18. Ruzzo A, Graziano F, Loupakis F, Rulli E, Canestrari E, Santini D, Catalano V, Ficarelli R, Maltese P, Bisonni R, Masi G, Schiavon G, Giordani P, Giustini L, Falcone A, Tonini G, Silva R, Mattioli R, Floriani I, Magnani M. Pharmacogenetic profiling in patients with advanced colorectal cancer treated with first-line FOLFOX-4 chemotherapy. J Clin Oncol 2007; 25: 1247-1254.

19. Paré L, Marcuello E, Altés A, del Río E, Sedano L, Salazar J, Cortés A, Barnadas A, Baiget M. Pharmacogenetic prediction of clinical outcome in advanced colorectal cancer patients receiving oxaliplatin/5-fluorouracil as first-line chemotherapy. Br J Cancer 2008; 99: 1050-1055.

20. Liang H, Liang J, Lv HY, Zhang K. Research on the clinical outcom e of advanced colorectal cancer treated $\mathrm{W}$ ith oxaliplatin influenced by ERCC1 gene polymorphism in peripheral blood. Chinese C linical Oncology 2008; 13: 4.

21. Martinez-Balibrea E, Abad A, Aranda E, Sastre J, Manzano JL, Díaz-Rubio E, Gómez-España A, Aparicio J, García T, Maestu I, Martínez-Cardús A, Ginés A, Guino E. Pharmacogenetic approach for capecitabine or 5-fluorouracil selection to be combined with oxaliplatin as first-line chemotherapy in advanced colorectal cancer. Eur J Cancer 2008; 44: 1229-1237.

22. Spindler KL, Andersen RF, Jensen LH, Ploen $J$ and Jakobsen A. EGF61 $\mathrm{A}>\mathrm{G}$ polymorphism as predictive marker of clinical outcome to first-line capecitabine and oxaliplatin in metastatic colorectal cancer. Ann Oncol 2010; 21: 535-539.

23. Chua W, Goldstein D, Lee CK, Dhillon H, Michael M, Mitchell P, Clarke SJ, Iacopetta B. Molecular markers of response and toxicity to FOLFOX chemotherapy in metastatic colorectal cancer. Br J Cancer 2009; 101: 998-1004.
24. Chang PM, Tzeng CH, Chen PM, Lin JK, Lin TC, Chen WS, Jiang JK, Wang HS, Wang WS. ERCC1 codon 118 C-->T polymorphism associated with ERCC1 expression and outcome of FOLFOX-4 treatment in Asian patients with metastatic colorectal carcinoma. Cancer Sci 2009; 100: 278-283.

25. Chen YC, Tzeng CH, Chen PM, Lin JK, Lin TC, Chen WS, Jiang JK, Wang HS, Wang WS. Influence of GSTP1 I105V polymorphism on cumulative neuropathy and outcome of FOLFOX-4 treatment in Asian patients with colorectal carcinoma. Cancer Sci 2010; 101: 530-535.

26. Liang J, Jiang T, Yao RY, Liu ZM, Lv HY, Qi WW. The combination of ERCC1 and XRCC1 gene polymorphisms better predicts clinical outcome to oxaliplatin-based chemotherapy in metastatic colorectal cancer. Cancer Chemother Pharmacol 2010; 66: 493-500.

27. Chai H, Pan J, Zhang X, Zhang X, Shen X, Li H, Zhang K, Yang C, Sheng H, Gao H. ERCC1 C118T associates with response to FOLFOX4 chemotherapy in colorectal cancer patients in Han Chinese. Int J Clin Exp Med 2012; 5: 186-194.

28. He WX, Li DM, ZHou WH, Deng JY, Wang YY. Association between polymorphism of ERCC 1 gene and sensitivity to platinumbased chemotherapy in advanced colon cancer. Guangdong Med J 2012; 33: 3.

29. van Huis-Tanja LH, Kweekel DM, Lu X, Franken K, Koopman M, Gelderblom H, Antonini NF, Punt CJ, Guchelaar HJ, van der Straaten T. Excision Repair Cross-Complementation group 1 (ERCC1) C118T SNP does not affect cellular response to oxaliplatin. Mutat Res Fundam Mol Mech Mutagen 2014; 759: 37-44.

30. Yin M, Yan J, Martinez-Balibrea E, Graziano F, Lenz HJ, Kim HJ, Robert J, Im SA, Wang WS, EtienneGrimaldi MC and Wei Q. ERCC1 and ERCC2 polymorphisms predict clinical outcomes of oxaliplatinbased chemotherapies in gastric and colorectal cancer: a systemic review and meta-analysis. Clin Cancer Res 2011; 17: 1632-1640.

31. Qian YY, Liu XY, Wu Q, Song X, Chen XF, Liu YQ, Pei D, Shen LZ, Shu YQ. The ERCC1 C118T polymorphism predicts clinical outcomes of colorectal cancer patients receiving oxaliplatin-based chemotherapy: a metaanalysis based on 22 studies. Asian Pac J Cancer Prev 2014; 15: 8383-8390.

\section{*Correspondence to}

Jian-Xun Lu

Department of Medical Oncology

The First People's Hospital of Nanning

PR China 